\title{
Características fonológicas de crianças com transtorno fonológico com e sem histórico de otite média
}

\author{
Phonological characteristics of children with phonological disorder with \\ and without otitis media history
}

\author{
Haydée Fiszbein Wertzner ${ }^{1}$, Luciana de Oliveira Pagan², Daniela Evaristo dos Santos Galea ${ }^{3}$, Ana Carolina \\ Camargo Salvatti Papp ${ }^{4}$
}

\begin{abstract}
RESUMO
Objetivo: Verificar o número de tipos, a ocorrência total e a média de processos fonológicos em crianças com transtorno fonológico com e sem o histórico de otite média. Métodos: A casuística foi composta de 44 crianças diagnosticadas com transtorno fonológico, metade com histórico de otite média e metade sem esse histórico do Laboratório de Investigação Fonoaudiológica em Fonologia do Curso de Fonoaudiologia da Faculdade de Medicina da USP-SP. Foram aplicadas as provas de nomeação e imitação (Wertzner, 2000) para a análise dos processos fonológicos. Resultados: No grupo com otite, o processo fonológico mais ocorrente na prova de nomeação foi o ensurdecimento de fricativas e, na imitação, a simplificação de líquidas. No grupo sem otite, a simplificação do encontro consonantal foi mais empregada na nomeação e, o ensurdecimento de fricativas na imitação. Somente a simplificação do encontro consonantal na prova de nomeação apresentou diferença entre os grupos. Não houve diferença estatística em relação à média de tipos de processos fonológicos. Conclusão: Apesar de a otite média estar presente em parte das crianças com o transtorno fonológico, a análise realizada não permitiu a identificação de marcadores lingüísticos que separassem os grupos estudados.
\end{abstract}

DESCRITORES: Transtornos do desenvolvimento de linguagem; Otite média; Desenvolvimento infantil; Linguagem infantil

\section{INTRODUÇÃO}

O transtorno fonológico é definido como uma alteração encontrada no sistema fonológico de um indivíduo e pode ser caracterizado por: substituições, omissões e ou distorções dos sons da fala. Essas alterações podem estar relacionadas às dificuldades com a organização das regras fonológicas da língua o que caracterizaria uma dificuldade cognitivo-lingüística, com a percepção auditiva dos sons e/ou com a produção dos mesmos ${ }^{(1-2)}$.

(1) Professora Associada, Livre Docente do Curso de Fonoaudiologia da Faculdade de Medicina da Universidade de São Paulo - USP - São Paulo (SP), Brasil.

(2) Pós-graduanda da Faculdade de Filosofia Letras e Ciências Humanas da Universidade de São Paulo - USP - São Paulo (SP), Brasil.

(3) Pós-graduanda da Faculdade de Filosofia Letras e Ciências Humanas da Universidade de São Paulo - USP - São Paulo (SP), Brasil.

(4) Pós-graduanda da Faculdade de Filosofia Letras e Ciências Humanas da Universidade de São Paulo - USP - São Paulo (SP), Brasil.

Trabalho realizado no Departamento de Fisioterapia, Fonoaudiologia e Terapia Ocupacional da Faculdade de Medicina da Universidade de São Paulo FMUSP - São Paulo (SP), Brasil.

Endereço para correspondência: Luciana de Oliveira Pagan. R. Dr. Nogueira Martins, 400/52, Saúde, São Paulo - SP, CEP 04143-020. E-mail: lopagan@ig.com.br

Recebido em: 28/9/2006; Aceito em: 8/2/2007
Há que se considerar que a fonologia envolve um repertório de fonemas que servem para indicar mudanças no significado das palavras, referindo-se assim, ao componente da linguagem que governa a maneira como os sons são produzidos na fala por meio de regras. Nesta concepção, a criança tem que aprender as regras fonológicas do sistema lingüístico de sua comunidade ${ }^{(3)}$. Assim, a criança domina gradualmente a percepção auditiva e a produção dos sons da fala. É por intermédio desta percepção que a criança, em um processo ativo, organiza as representações internas a respeito da língua materna para produzir os sons de determinada língua.

No período em que a criança desenvolve suas habilidades perceptivas, adquire um vasto inventário de elementos fonéticos, perde uma variedade de processos fonológicos de simplificação e adquire um sistema fonológico de contrastes ${ }^{(1,4)}$.

Um modelo de análise fonológica, muito empregado na descrição do sistema fonológico, é o dos processos fonológicos. Esses se referem à simplificação de regras fonológicas que envolvem um grupo ou uma seqüência de sons da fala, e que ocorrem durante o desenvolvimento de linguagem. Assim, a criança deve eliminar os processos que não fazem parte da linguagem adulta ${ }^{(1,4-7)}$. Se uma criança apresentar estes processos fonológicos além da idade esperada, é considerada como portadora de transtorno fonológico. 
Os processos fonológicos podem ser divididos em três categorias: de estrutura silábica, substituição e assimilação ${ }^{(1,4)}$. Os processos de estrutura silábica são: simplificação da consoante final, simplificação do encontro consonantal, eliminação da sílaba átona, reduplicação e metátese. Os processos de substituição são: plosivação de fricativas, simplificação de líquidas, vocalização, despalatalização, frontalização de velar, desafricação, eliminação da estridência. Por fim, os processos de assimilação englobam as assimilações labial, velar, nasal e sonora.

A supressão ou o desaparecimento de determinado processo ocorre gradativamente com a sua aplicação restrita a apenas alguns fonemas ou posições específicas dentro da palavra $^{(4)}$.

Apesar de ser possível a caracterização do transtorno fonológico, ainda não se consegue determinar sua causa, embora se verifiquem causas correlatas o que sugere a existência de diferentes tipos de transtorno fonológico de causa desconhecida $^{(8)}$.

Existem três classificações diferentes para o transtorno fonológico: a descrição lingüística, os processos neurolinguiísticos e a etiologia. Pela descrição lingüística realizada a partir dos vários modelos teóricos é possível verificar quais princípios fonéticos e fonológicos fundamentam os erros encontrados na fala, bem como nas regras empregadas obtendo-se, portanto, a indicação dos padrões de $\operatorname{erros}^{(8)}$.

Pela etiologia, é possível observar quais fatores genéti$\cos$ e ambientais constituem risco para o transtorno fonológico. Para esse tipo de estudo são realizadas pesquisas epidemiológicas e de risco e normalização do transtorno, indicando a sua causa.

A grande dificuldade em estabelecer possíveis marcadores diagnósticos que possam ajudar na identificação e no tratamento precoces de crianças com atraso de fala é relatada em diversos estudos ${ }^{(9-12)}$. Estes marcadores são definidos como sendo características mensuráveis, usadas para classificar como os indivíduos podem ser afetados ou não por um tipo específico de transtorno. Embora eles possam fornecer informações úteis no que diz respeito à explicação sobre a natureza do transtorno, seu primeiro objetivo é ser uma ferramenta de classificação diagnóstica.

Desta forma, são sugeridos cinco subgrupos com diferentes etiologias que mostrem substituições e eliminações que não sejam adequadas à idade, além de dificuldades acadêmicas e problemas sociais ${ }^{(8-9)}$. Os cinco subgrupos são:

1- atraso de fala genético: possibilidade de ser o responsável por cerca de 40 a $60 \%$ dos casos com transtorno de linguagem expressiva entre três e cinco anos de idade;

2- atraso de fala decorrente de episódios de otite média de repetição: representa $30 \%$ das crianças com atraso de fala. Crianças com esse histórico apresentam idade inadequada para as omissões e as substituições, alto risco de transtorno de fala expressiva e dificuldades acadêmicas;

3- atraso de fala, acompanhado de Developmental Apraxia of Speech (DAS - Apraxia de Desenvolvimento de Fala): estima-se que seja o responsável por cerca de 5\% dos casos de atraso de fala. Há a presença de evidências de envolvimento motor oral sem justificativa, com alto ris- co de transtorno de fala expressiva;

4- atraso de fala envolvendo o desenvolvimento psicossocial: ocorre em menos de $5 \%$ da população com transtorno fonológico. Requer acompanhamento psicossocial ou psicopedagógico;

5- erros residuais: apresentam apenas histórias de distorções em fricativas/africadas e/ou líquidas de três a cinco anos e/ou seis a oito anos, sem história de atraso de fala e transtorno de linguagem.

A identificação de possíveis marcadores que auxiliem no diagnóstico dos subtipos do transtorno fonológico parece ser muito apropriada, já que os avanços tecnológicos permitem pensar a respeito da origem e da natureza deste tipo de transtorno $^{(11)}$

Dentre esses cinco subtipos citados, certamente o mais estudado e mais contraditório é o que relaciona os atrasos de fala a episódios de OM recorrente durante a primeira infância.

A otite média é uma inflamação na orelha média freqüentemente associada a um aumento de fluido infectado ou não. É uma doença multifatorial, incluindo vários fatores etiológicos, tais como: disfunção da tuba auditiva e infecções de vias aéreas superiores. Os principais fatores de risco para otites são: baixo peso ao nascimento, a idade precoce do primeiro episódio de otite, pois há a tendência dos episódios se repetirem, histórico de alergia, congestão nasal, presença de bactérias na naso-faringe e histórico familiar.

A otite média é de grande prevalência na primeira infância, decrescendo com a idade ${ }^{(13-14)}$. Segundo dados da American Speech and Hearing Association ${ }^{(15)}$, 50\% das crianças com um ano de idade já apresentaram pelo menos um episódio de otite.

Esse quadro é muito comum em crianças pequenas devido à maior horizontalização da tuba auditiva, o que favorece a repetição dos episódios de otite média. A perda auditiva causada pela otite ocorre devido ao fluido acumulado na orelha média, que dificulta a transmissão das vibrações sonoras através da cadeia ossicular, causando perda de energia sonora. Como resultado, tem-se uma perda auditiva de grau leve ou até mesmo moderado, o que é suficiente para tornar alguns sons inaudíveis alterando assim, a percepção da fala ${ }^{(13,16)}$.

Um consenso geral sobre a otite média é o de que ela provoca uma perda auditiva condutiva temporária. A relação entre o desenvolvimento da linguagem e o histórico de otite média em crianças vem sendo bastante estudada, mas, a extensão correta dessa relação, ainda permanece como um grande ponto de debate ${ }^{(17)}$

Alguns estudos relataram diferenças significantes entre as alterações fonológicas apresentadas por crianças com e sem histórico de otite média. Em geral, as crianças com esse histórico apresentaram desempenhos mais baixos em testes de percepção e processamento auditivo, grande incidência de problemas acadêmicos, especialmente alterações de leitura e escrita ${ }^{(18-21)}$. Estes autores acreditam que a perda auditiva temporária que acompanha a otite torna alguns sons inaudíveis e, apesar de ser transitória, altera a qualidade de percepção dos sons uma vez que o sinal auditivo pode resultar em um sinal incompleto ou inconsistente, o que induz a uma alteração na codificação das distinções fonéticas ${ }^{(22)}$. 
Outros estudos, entretanto, mostram que a perda auditiva ocasionada pela otite não provoca um grande prejuízo no aprendizado da linguagem ${ }^{(17,23-25)}$. Estes pesquisadores acreditam que a otite média não tem efeito sobre a linguagem, baseando-se no fato de que a perda auditiva condutiva que ocorre varia de leve a moderada (perda auditiva flutuante) durante os episódios de otite. Assim, após estas ocorrências, a audição volta à normalidade e os efeitos da otite são compensados pela audição normal.

$\mathrm{O}$ risco de atraso de fala decorrente de episódios de otite média é baseado na falha perceptual que ocorre no estabelecimento adequado da fonologia da língua durante o período de aquisição da linguagem, ao contrário dos riscos associados às dificuldades articulatórias e/ou cognitivas ${ }^{(12)}$. Em seus estudos, os autores observaram que a fala de crianças com atraso de fala e presença de histórico de otite média parece ser notavelmente mais ininteligível do que as de crianças com atraso de fala, mas sem o histórico da otite média. Para isso existiriam duas explicações possíveis. A primeira delas está relacionada à perspectiva fonético-acústica que indica uma falha na discriminação, no armazenamento e na reprodução de muitos contrastes acústicos necessários ao ouvinte para a compreensão do significado. Estas dificuldades perceptuais referem-se aos sinais auditivos que surgem com falhas durante os episódios de otite média. A segunda explicação é a de que as perdas auditivas flutuantes provocam um efeito difuso nas habilidades cognitivas e lingüísticas, afetando tanto a fala quanto a percepção dos fonemas. A primeira alternativa é mais consistente com a possibilidade de identificação de um marcador diagnóstico, entretanto, existem muitas considerações teóricas que também fornecem a base para a segunda.

$\mathrm{O}$ aspecto fonológico da linguagem é um dos mais afetados pela perda auditiva condutiva flutuante. A perda auditiva condutiva flutuante tipicamente acompanhada de otite média pode resultar na aquisição inadequada dos sons da fala ${ }^{(26)}$. Alguns estudos indicaram que uma perda auditiva condutiva pode afetar a produção da fala mais diretamente do que as habilidades de linguagem receptiva ${ }^{(20,27)}$. Crianças com histórico de otite média apresentaram inventário de consoantes produzidas relativamente menor do que o número de vogais $^{(19)}$.

Em uma pesquisa, foi realizado um estudo longitudinal com crianças com transtorno fonológico ${ }^{(21)}$. No primeiro estudo, os autores não encontraram diferenças no desempenho fonológico entre os grupos com e sem história positiva de otite média com efusão. Porém, no segundo estudo, essa diferença foi registrada, indicando a necessidade de um modelo multifatorial que relacione a otite média de repetição ao transtorno fonológico tardio.

Muitos dos possíveis marcadores dependem da descrição lingüística da fonologia e, portanto, são individuais para cada língua. Por isso, estudos para o português brasileiro precisam ser realizados para identificá-los.

Assim, a pesquisa teve por objetivo verificar os tipos, a ocorrência total e a média de uso de processos fonológicos em crianças com transtorno fonológico, com e sem o histórico de otite média.

\section{MÉTODOS}

A presente pesquisa enquadra-se no tipo observacional, transversal, retrospectiva e comparativa de grupos paralelos. Não expõe os indivíduos a riscos.

Essa pesquisa foi aprovada pela Comissão de Ética para Análise de Projetos de Pesquisa - CAPPesq da Diretoria Clínica do Hospital das Clínicas e da Faculdade de Medicina da Universidade de São Paulo (protocolo 286/99). O Consentimento Livre e Esclarecido foi assinado pelos responsáveis.

Foram analisados os protocolos de 109 sujeitos, sendo 85 deles com histórico de otite média (OM) e infecções de vias aéreas superiores (IVAS) e 22, sem histórico de ambos os gêneros. No grupo de sujeitos com diagnóstico de transtorno fonológico com histórico, dentre os 85 sujeitos com histórico, apenas 22 apresentaram apenas histórico de otite média, sendo 15 do gênero masculino e sete do gênero feminino com idade entre 4:3 e 12:5 anos. No grupo de sujeitos com diagnóstico de transtorno fonológico sem histórico foram analisados os 22, sendo 14 do gênero masculino e oito do gênero feminino com idade entre 5:2 e 12:6 anos.

As crianças de ambos os grupos eram pacientes do Laboratório de Investigação Fonoaudiológica em Fonologia (LIF) do Curso de Fonoaudiologia da Faculdade de Medicina da Universidade de São Paulo, situado no prédio do Departamento de Fisioterapia, Fonoaudiologia e Terapia Ocupacional da FMUSP, no campus da Cidade Universitária.

Como critério de inclusão as crianças do grupo com otite deveriam ter o diagnóstico de transtorno fonológico, não ter passado por terapia fonoaudiológica anteriormente, apresentar apenas histórico de OM. Para as crianças do grupo sem otite o critério adotado foi o mesmo, mas as crianças não poderiam ter nem histórico de OM nem de IVAS.

Após a assinatura do termo de consentimento livre e esclarecido pelos responsáveis, todas as crianças passaram por avaliação fonoaudiológica para diagnóstico de transtorno fonológico.

Para a pesquisa foi utilizado um roteiro de anamnese e as provas de fonologia do Teste de Linguagem Infantil ABFW ${ }^{(28)}$.

Durante a aplicação das provas a avaliadora sentou-se em frente ao sujeito, sendo que o material e as folhas de registro ficaram sobre a mesa. O gravador foi posicionado de modo que o microfone ficasse direcionado e próximo à criança, para evitar interferências na gravação, possibilitando uma melhor transcrição fonética das provas. A filmadora foi colocada em um tripé permitindo o foco adequado do sujeito, o que favoreceu a observação de sua produção articulatória, minimizando a possibilidade de dificuldades na transcrição fonética.

Após a aplicação das provas de Fonologia, as mesmas foram transcritas foneticamente nas folhas de registro propostas nas provas de Fonologia do ABFW (Wertzner, 2000) e os processos fonológicos apresentados foram classificados.

A análise estatística dos dados utilizou o Teste de Friedman (n sig 0.05), o Teste t de Student para Dados Pareados (n sig 0.05), o Teste t de Student controlado pelo Teste de Levene para Igualdade de Variâncias e o Teste dos Postos Sinalizados de Wilcoxon. 


\section{RESULTADOS}

Primeiramente, foram analisados os processos fonológicos de maior ocorrência nos grupos de crianças com otite e sem otite tanto na nomeação como na imitação.

Quanto ao grupo de crianças com otite, a Figura 1 mostra que o processo fonológico de maior ocorrência na prova de nomeação foi o de ensurdecimento de fricativas, seguido do ensurdecimento de plosivas e de simplificação de líquidas.

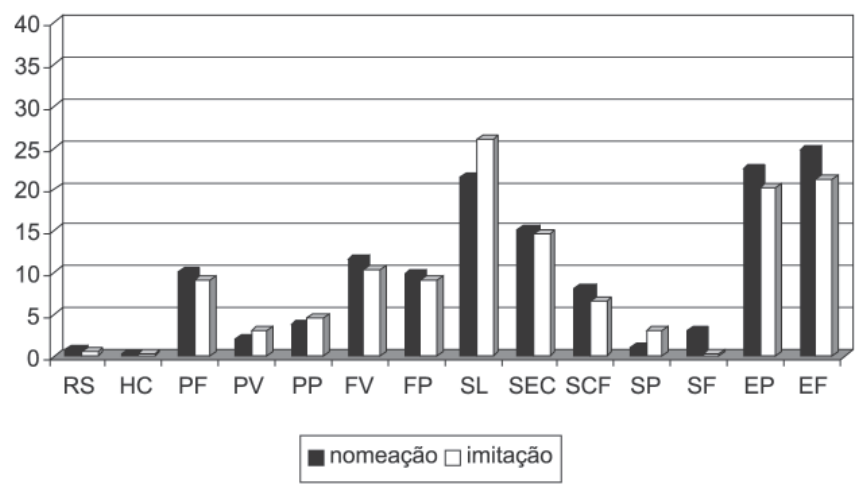

Legenda: $\mathrm{RS}=$ redução de sílaba, $\mathrm{HC}=$ harmonia consonantal, $\mathrm{PF}=$ plosivação de fricativas, $\mathrm{FV}$ = frontalização de velar, $\mathrm{PV}=$ posteriorização para velar, $\mathrm{PP}=$ posteriorização para palatal, $\mathrm{FP}=$ frontalização de palatal, $\mathrm{SL}=$ simplificação de líquidas, $\mathrm{SEC}=$ simplificação do encontro consonantal, SCF = simplificação da consoante final, SP = sonorização de plosiva, $\mathrm{SF}=$ sonorização de fricativa, $\mathrm{EP}=$ ensurdecimento de plosiva, $\mathrm{EF}=$ ensurdecimento de fricativa.

Figura 1. Média de ocorrência de processos fonológicos na prova de nomeação e imitação para os sujeitos com otite

Aplicou-se o Teste de Friedman (n sig 0.05) para comparar as ocorrências de todos os processos fonológicos simultaneamente e observou-se $\mathrm{p}<0,001$. Assim, foi aplicado o Teste dos Postos Sinalizados de Wilcoxon, para identificar as diferenças entre os pares de processos fonológicos.

$\mathrm{O}$ processo mais ocorrente, ensurdecimento de fricativas, apresentou ocorrência estatisticamente igual aos processos de: ensurdecimento de plosivas $(\mathrm{p}=0.649)$, simplificação de líquidas ( $\mathrm{p}=0.845)$, simplificação do encontro consonantal $(\mathrm{p}=0.480)$, frontalização de velar $(\mathrm{p}=0.139)$, plosivação de fricativas $(\mathrm{p}=0.248)$, frontalização de palatal $(\mathrm{p}=0.086)$ e simplificação da consoante final $(\mathrm{p}=0.059)$.

$\mathrm{Na}$ imitação, as crianças com otite tiveram uso maior do processo de simplificação de líquidas seguido de ensurdecimento de fricativas, ensurdecimento de plosivas e simplificação do encontro consonantal (Figura 1).

Nesta prova, também foi aplicado o Teste de Friedman (n sig 0.05) para a comparação das ocorrências de todos os processos fonológicos simultaneamente, sendo observado $\mathrm{p}<0,001$.

Aplicando-se o Teste dos Postos Sinalizados de Wilcoxon, observou-se que o processo mais ocorrente, simplificação de líquidas, tem ocorrência estatisticamente igual aos de: ensurdecimento de fricativas $(\mathrm{p}=0.309)$, ensurdecimento de plosivas $(\mathrm{p}=0.379)$ e simplificação do encontro consonantal $(\mathrm{p}=0.201)$. Os demais processos são estatisticamente diferentes da simplificação de líquida.

Em relação ao grupo de crianças sem otite, a Figura 2 mostra que o processo fonológico de maior ocorrência na prova de nomeação foi o de simplificação do encontro consonantal, seguido de ensurdecimento de fricativas, ensurdecimento de plosivas e simplificação de líquidas.

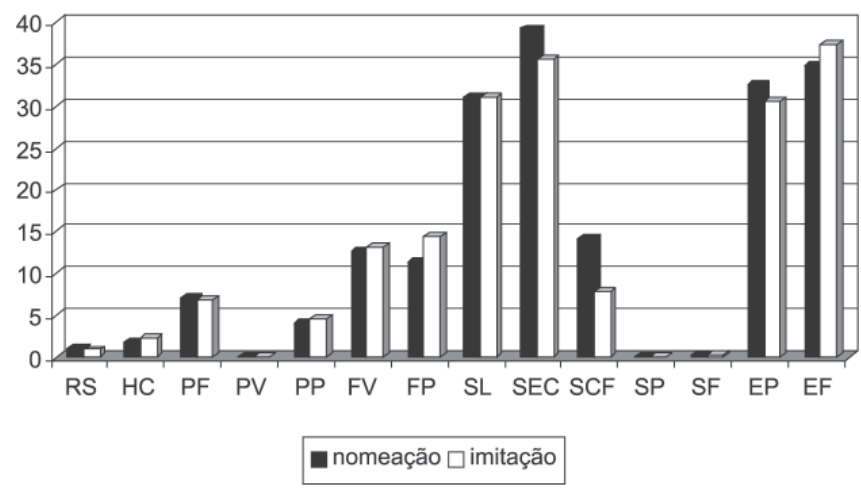

Legenda: $\mathrm{RS}=$ redução de sílaba, $\mathrm{HC}=$ harmonia consonantal, $\mathrm{PF}=$ plosivação de fricativas, $\mathrm{FV}$ = frontalização de velar, $\mathrm{PV}=$ posteriorização para velar, $\mathrm{PP}=$ posteriorização para palatal, $\mathrm{FP}=$ frontalização de palatal, SL = simplificação de líquidas, SEC = simplificação do encontro consonantal, SCF = simplificação da consoante final, SP = sonorização de plosiva, SF = sonorização de fricativa, EP = ensurdecimento de plosiva, EF = ensurdecimento de fricativa.

Figura 2. Média de ocorrência de processos fonológicos na prova de nomeação e imitação para os sujeitos sem otite

Utilizando o Teste de Friedman (n sig 0.05), observou-se $\mathrm{p}<0,001$ para a comparação das ocorrências de todos os processos fonológicos simultaneamente.

Aplicando-se o Teste dos Postos Sinalizados de Wilcoxon, observou-se que o processo mais ocorrente, simplificação do encontro consonantal, é estatisticamente igual aos de: ensurdecimento de fricativas $(\mathrm{p}=0.619)$, ensurdecimento de plosivas ( $\mathrm{p}=0.459$ ) e simplificação de líquidas $(\mathrm{p}=0.444)$. Os demais processos são estatisticamente diferentes da simplificação do encontro consonantal.

$\mathrm{Na}$ imitação (Figura 2), o processo mais usado foi o ensurdecimento de fricativas, seguido de simplificação do encontro consonantal, simplificação de líquidas e ensurdecimento de plosivas.

No Teste de Friedman (n sig 0.05), observou-se p $<0,001$ para a comparação das ocorrências de todos os processos fonológicos simultaneamente.

Porém, aplicando-se o Teste dos Postos Sinalizados de Wilcoxon, observou-se que o processo mais ocorrente, ensurdecimento de fricativas, é estatisticamente igual aos de: simplificação do encontro consonantal $(\mathrm{p}=0.913)$, simplificação de líquidas $(\mathrm{p}=0.542)$, ensurdecimento de plosivas $(\mathrm{p}=0119)$ e frontalização de palatal $(\mathrm{p}=0.123)$. Os demais processos são estatisticamente diferentes do ensurdecimento de fricativas.

Com o intuito de se analisar os processos fonológicos em cada um dos grupos, comparando os resultados da prova de 
nomeação e imitação, foi aplicado o Teste t de Student para Dados Pareados (n sig 0.05).

Para o grupo de sujeitos com otite, não houve diferença estatisticamente significante ao se comparar cada um dos processos fonológicos nas duas provas. Porém, no grupo de sujeitos sem otite, houve diferença entre a prova de imitação e nomeação quanto ao processo de simplificação da consoante final ( $\mathrm{p}=0.032$ ), sendo que os sujeitos tiveram ocorrência maior deste processo na prova de nomeação.

Foi realizada comparação de cada um dos processos fonológicos entre os grupos em cada uma das provas, aplicando-se o Teste t de Student controlado pelo Teste de Levene para Igualdade de Variâncias.

Na prova de nomeação, houve diferença estatisticamente significante somente para o processo de simplificação do encontro consonantal $(\mathrm{p}=0.034)$, sendo que o grupo de crianças sem otite obteve média de ocorrência maior que o grupo com otite.

Na prova de imitação, todas as médias de ocorrência dos processos fonológicos foram estatisticamente iguais entre os dois grupos.

Outra análise realizada refere-se à média de tipos de processos fonológicos intragrupos e intergrupos para as provas de nomeação e imitação. O grupo de crianças com otite apresentou menor número de tipos diferentes de processos fonológicos que as crianças sem otite (Figura 3). Observa-se também que ambos os grupos apresentaram maior número de tipos diferentes de processos na prova de nomeação.

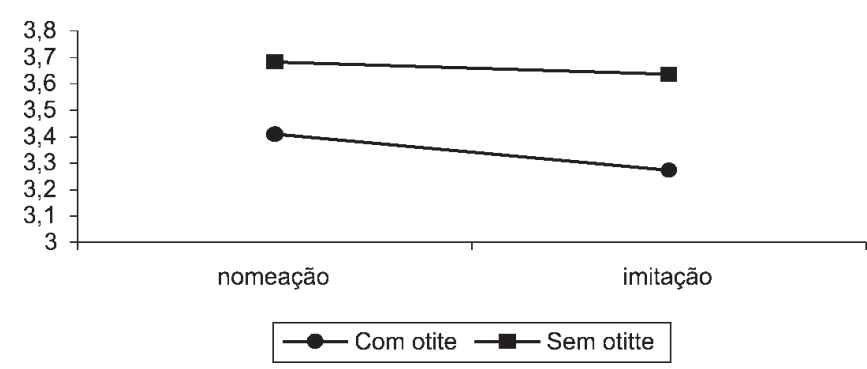

Figura 3. Comparação entre a média de tipo de processos fonológicos nos grupos e em cada prova

O Teste t de Student, controlado pelo Teste de Levene para Igualdade de Variâncias (n sig. 0.05), indicou que não há diferença estatisticamente significante entre os grupos, tanto na nomeação $(\mathrm{p}=0.655)$ como na imitação $(\mathrm{p}=0.579)$.

O Teste t de Student para dados Pareados (n sig 0.05) mostrou que não há diferença em relação à média de tipo de processos fonológicos entre as provas no grupo de crianças com otite $(\mathrm{p}=0.701)$ e sem otite $(\mathrm{p}=0.771)$.

\section{DISCUSSÃO}

Como foi apontado, o transtorno fonológico não tem causa definida e, por isso, sua etiologia é bastante discutida. Estudos recentes apontam que o transtorno fonológico pode ser classificado em vários subtipos. Um dos subtipos tem como uma de suas possíveis causas, o histórico de otite média durante a primeira infância. Apoiando-se nesta hipótese, a pre- sente pesquisa procurou verificar fatores que indicassem um marcador fonológico que diferenciasse o grupo de crianças com transtorno fonológico apresentando o histórico de otite média das crianças do grupo sem esse histórico.

Observou-se que, na maioria dos casos, os processos fonológicos apresentados pelos dois grupos de crianças estudados foram semelhantes. Entretanto, pode-se observar que esses foram também os mais notados em sujeitos com transtorno fonológico independentemente de terem, ou não, episódios de otite média em seu histórico de vida.

Nas crianças desse estudo, os processos fonológicos mais comumente apresentados foram: Simplificação do Encontro Consonantal (SEC), Simplificação da Consoante Final (SCF), Simplificação de Líquida (SL), Ensurdecimento de Plosiva (EP), Ensurdecimento de Fricativa (EF) e Frontalização da Palatal (FP). Estes achados corroboram os resultados encontrados em pesquisas realizadas com crianças falantes do português apresentando transtorno fonológico ${ }^{(29-30)}$.

Embora não se tenha encontrado diferença significante entre as ocorrências dos processos fonológicos nos dois grupos estudados, nota-se que o grupo de crianças com otite teve maior ocorrência na prova de nomeação de ensurdecimento de fricativas, seguido do ensurdecimento de plosivas e de simplificação de líquidas. Já na imitação, apresentaram o processo de simplificação de líquidas seguido de ensurdecimento de fricativas, ensurdecimento de plosivas e simplificação do encontro consonantal. Isso poderia ser um indicativo de que com o auxilio da produção a criança perceba auditivamente a diferença dos sons sonoros e, na medida em que não tem dificuldades de produzir a sonoridade, consegue uma emissão correta do som.

Em relação ao grupo de crianças sem otite, a maior ocorrência na prova de nomeação foi a simplificação do encontro consonantal, seguida de ensurdecimento de fricativas, ensurdecimento de plosivas e simplificação de líquidas. Na imitação o processo mais usado foi o ensurdecimento de fricativas, seguido de simplificação do encontro consonantal, simplificação de líquidas e ensurdecimento de plosivas. Da mesma maneira que no outro grupo, a criança sem otite parece se beneficiar da estimulação para a produção do encontro consonantal, mas não para a emissão da sonoridade o que pode indicar maior dificuldade para essa produção.

De forma geral, em ambos os grupos participantes desse estudo, os processos produzidos pelo maior número de sujeitos foram os de ensurdecimento de plosivas e de fricativas, mas cabe ressaltar que estes processos fonológicos são dos mais ocorrentes na população com transtorno fonológico ${ }^{(30)}$. Esses achados mostram que para o Português Brasileiro a classe de sons que estaria mais susceptível a ser produzida de maneira errada, é diferente da apresentada para os falantes do inglês norte-americano. Estudos apontam que o processo fonológico mais observado em crianças que apresentaram em seu histórico de vida episódios de otite média é o de posteriorização das obstruintes, ou seja, articular plosivas e fricativas, posicionando a língua de um modo mais posterior $^{(8,10-11)}$.

Em ambos os grupos do estudo, a média de diferentes tipos de processos apresentados pelos sujeitos foi de três. Os 
estudos indicaram que a maior parte de crianças analisadas em seu estudo usou três processos fonológicos diferentes mostrando que, em geral, as crianças com transtorno fonológico tendem a apresentar poucos tipos de processos, mas com grande ocorrência ${ }^{(30)}$. Portanto, no que se refere aos tipos de processos fonológicos usados, o grupo com histórico de otite média não apresentou diferenças do grupo sem este histórico e apresentou resultados similares às crianças com transtorno fonológico, independentemente de apresentar ou não queixa de otite.

Os processos determinantes para caracterizar o transtorno fonológico são: ensurdecimento de plosivas, ensurdecimento de fricativas, frontalização da palatal, simplificação do encontro consonantal e simplificação de líquidas ${ }^{(31)}$. Os resultados do presente estudo mostram que esses foram os processos mais ocorrentes, também, em ambos os grupos, e que são estatisticamente iguais, não diferenciando, portanto, quanto às características fonológicas o grupo com histórico de otite média.

O fato das crianças de ambos os grupos apresentarem maior número de tipos diferentes de processos na prova de nomeação, evidencia que a sua grande dificuldade esteja localizada na organização lingüística-cognitiva das regras do sistema fonológico da língua, isto é, na população estudada parece haver uma característica predominante de sujeitos com dificuldade em compreender e usar as regras fonológicas, do que em produzir os sons ${ }^{(32)}$.

Portanto, os resultados desse estudo, assim como os observados por outros autores ${ }^{(17,23-25)}$ não demonstraram diferenças fonológicas que pudessem corroborar com a hipótese da existência de marcadores lingüísticos que diferenciem o desempenho das crianças com histórico de otite média.

A réplica desse tipo de estudo é importante, pois os marcadores fonológicos poderiam auxiliar o fonoaudiólogo clínico tanto no momento do diagnóstico como no planejamento da intervenção terapêutica. Destaca-se, portanto, que a possibilidade de identificação do fator causal correlacionado ao transtorno fonológico facilitaria a intervenção.

\section{CONCLUSÃO}

Os resultados desta pesquisa mostram que, apesar da otite média ter uma relação com o transtorno fonológico, a análise fonológica realizada não permitiu a identificação de marcadores lingüísticos que separassem os dois grupos estudados.

\begin{abstract}
Purpose: To verify the number of types, the total occurrence and the mean of phonological processes in phonologically disordered children with and without otitis background. Methods: The subjects were 44 children diagnosed with phonological disorder, half of them with otitis background and half without any history of otitis, from the Laboratory of Phonology of the Speech and Language Pathology Course of the Medical School USP-SP. The imitation and picture naming tests (Wertzner, 2000) were carried out in order to analyze the phonological processes. Results: In the otitis group, the most occurring phonological process in the imitation test was the fricative devoicing and in the picture naming, the liquid simplification. In the group of children with no otitis background, the cluster reduction and the fricative devoicing were the most used processes in the picture naming and in the imitation tests, respectively. Only the cluster reduction in the picture naming test had differences between both groups. There was no statistical difference regarding the mean of phonological processes types. Conclusion: Although some children with phonological disorder might present otitis media, the analysis did not allow the identification of a linguistic marker that can differentiate children with and without otitis background.
\end{abstract}

KEYWORDS: Language development disorders; Otitis media; Child development; Child language

\title{
REFERÊNCIAS
}

1. Ingram D. Phonological disability in children. London: Edward Arold; 1976.

2. American Psychiatric Association.diagnostic and statistical manual of mental disorders DSM-IV. 4th ed. Washington DC: APA; 1994.

3. Fey ME. Articulation and phonology: inextricable constructs in speech pathology. Lang Speech Hear Serv Sch. 1992;23:225-32.

4. Stoel-Gammon C, Dunn C. Normal and disordered phonology in children. Austin: Pro-Ed; 1985.

5. Edwards ML. Clinical forum: Phonological assessment and treatment in support of phonological processes. Lang Speech Hear Serv Sch. 1992;23:233-40.

6. Wertzner HF. Estudo da aquisição do sistema fonológico: o uso de processos fonológicos em crianças de três a sete anos. PróFono.1995;7(1):21-6.
7. Galea DES. Análise do sistema fonológico em crianças de 2;1 a 3;0 anos de idade [tese]. São Paulo: Faculdade de Filosofia Letras e Ciências Humanas da Universidade de São Paulo; 2003.

8. Shriberg LD. Diagnostic classification of five subtypes of childhood speech sound disorders (SSD) of currently unknown origin. Paper presented at the International Association of Logopedics and Phoniatrics Congress, Brisbane, Australia; 2004

9. Shriberg LD. Epidemiologic and Diagnostic Profiles for Five Developmental Phonological Disorders. Seminar presented at the Annual Convention or the American Speech-Language-Hearing Association. San Francisco; 1999.

10. Shriberg LD. Classification and misclassification of child speech sound disorders. Paper presented at the America Speech-Language-Hearing Association Convention, Atlanta, GA, November 2002. 
11. Shriberg LD, Kent RD, Karlsson HB, McSweeny JL, Nadler CJ, Brown RL. A diagnostic marker for speech delay associated with otitis media with effusion: backing of obstruents. Clin Linguist Phon. 2003;17(7):529-47.

12. Shriberg LD, Flipsen P Jr, Kwiatkowski J, McSweeny JL. A diagnostic marker for speech delay associated with otitis media with effusion: the intelligibility-speech gap. Clin Linguist Phon. 2003;17(7):507-28.

13. Teele DW, Klein JO, Chase C, Menyuk P, Rosner BA. Otitis media in infancy and intellectual ability, school achievement, speech, and language at age 7 years. Greater Boston Otitis Media Study Group. J Infect Dis. 1990;162(3):685-94.

14. Paradise JL. Otitis media and child development: should we worry? Pediatr Infect Dis J. 1998;17(11):1076-83; discussion 1099-100.

15. American Speech and Hearing Association. Otitis media, hearing and language development [text on the Internet] [cited 2006 Jul 23]. Available from http://www.asha.org/consumers/brochures/ otitis_media.htm ; 2000.

16. Schochat E, organizador. Processamento auditivo: atualidades em Fonoaudiologia. São Paulo: Lovise; 1996.

17. Casby MW. Otitis media and language development: a meta-analysis. Am J Speech Lang Pathol. 2001;10(1):65-80.

18. Shriberg LD, Smith AJ. Phonological correlates of middle-ear involvement in speech-delayed children: a methodological note. J Speech Hear Res. 1983;26(2):293-7.

19. Abraham SS, Wallace IF, Gravel JS. Early otitis media and phonological development at age 2 years. Laryngoscope. 1996;106(6):727-32.

20. Mody M, Schwartz RG, Gravel JS, Ruben RJ. Speech perception and verbal memory in children with and without histories of otitis media. J Speech Lang Hear Res. 1999;42(5):1069-79.

21. Shriberg LD, Flipsen P Jr, Thielke H, Kwiatkowski J, Kertoy MK, Katcher ML, et al. Risk for speech disorder associated with early recurrent otitis media with effusion: two retrospective studies. J Speech Lang Hear Res. 2000;43(1):79-99.

22. Gravel JS, Wallace IF. Listening and language at 4 years of age: effects of early otitis media. J Speech Hear Res. 1992;35(3):588-95.
23. Roberts JE, Burchinal MR, Davis BP, Collier AM, Henderson FW. Otitis media in early childhood and later language. J Speech Hear Res. 1991;34(5):1158-68.

24. Harsten G, Nettelbladt U, Schalen L, Kalm O, Prellner K. Language development in children with recurrent acute otitis media during the first three years of life. Follow-up study from birth to seven years of age. J Laryngol Otol. 1993;107(5):407-12.

25. Wertzner HF, Rosal CAR, Pagan LO. Ocorrência de otite média e infecções de vias aéreas superiores em crianças com distúrbio fonológico. Rev Soc Bras Fonoaudiol. 2002;7(1):31-7.

26. Paden EP, Novak MA, Beiter AL. Predictors of phonologic inadequacy in young children prone to otitis media. J Speech Hear Disord. 1987;52(3):232-42.

27. Shriberg LD, Friel-Patti S, Flipsen P Jr, Brown RL. Otitis media, fluctuant hearing loss, and speech-language outcomes: a preliminary structural equation model. J Speech Lang Hear Res. 2000;43(1):10020.

28. Wertzner HF. Fonologia. In: Andrade CRF, Befi-Lopes DM, Fernandes FDM, Wertzner HF. ABFW: Teste de linguagem infantil nas áreas de Fonologia, Vocabulário, Fluência e Pragmática. São Paulo: Pró-Fono; 2000 .

29. Oliveira MMF, Wertzner HF. Estudo do distúrbio fonológico em crianças. Rev Soc Bras Fonoaudiol. 2000;7:68-75.

30. Wertzner HF, Oliveira MMF. Semelhanças entre os sujeitos com distúrbio fonológico. Pró-Fono. 2002;14(2):143-52.

31. Wertzner HF, Herrero SF, Ideriha PN, Pires SCF. Classificação do distúrbio fonológico por meio de duas medidas de análise: porcentagem de consoantes corretas (PCC) e índice de ocorrências dos processos (PDI). Pró-Fono. 2001;13(1):90-7.

32. Wertzner HF, Papp ACCS, Galea DES. Provas de nomeação e imitação como instrumentos de diagnóstico do transtorno fonológico. Pró-Fono. 2006;18(3):303-12. 